Historic, Archive Document

Do not assume content reflects current scientific knowledge, policies, or practices. 



\section{Grade-Count List}

\section{ROSE BUSEIES}

For Immediate Shipment

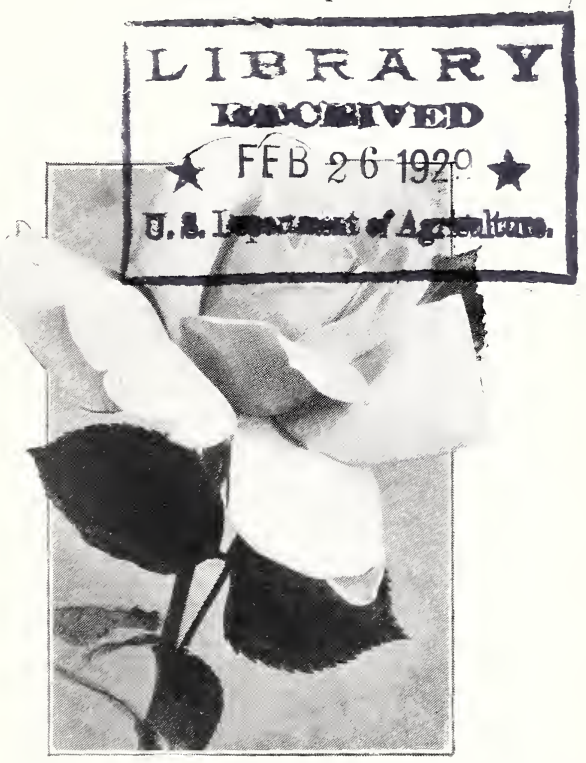

Howard Rose Company

Hemet, California

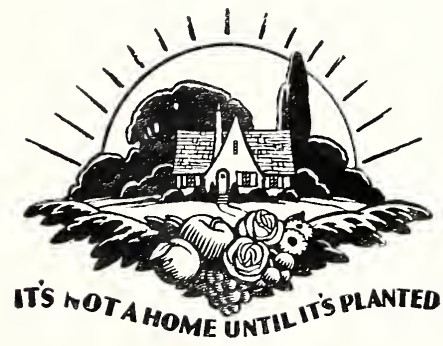




\section{PRICES-FEBRUARY 22, 1929}

\section{Sixth List}

Grade No. 1-Teas, Hybrid Teas and Polyanthas, 18 inches and up. Climbers and Hybrid Perpetuals, 24 inches and up. Three or more branches.

Grade No. $11 / 2$ or Medium-Teas, Hybrid Teas and Polyarthas, 14 inches and up. Climbers and Hybrid Perpetuals, 18 inches and up. Two or more branches.

Order in multiples of TEN. Ten or more at these prices

$$
\text { Lot } A \text { No. } 1 \frac{1-25 c}{2-15 c}
$$

No. 1

140 *Amelia d' Bethune, Per, (1923), coral....... 200

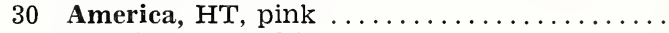

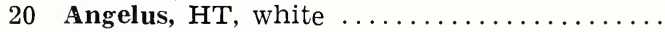

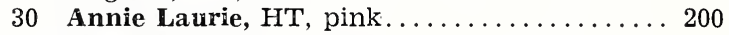

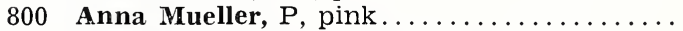

310 Antoine Rivoire, HT, rose-flesh. . . . . . . . 100

100 Audrey, HT, (1922), deep crimson........ 110

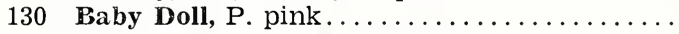

360 Baby Pink (Special, 20 cents) $\ldots \ldots \ldots \ldots \ldots$

70 Bessy Brown, HT, creamy-white......... 60

40 Betty, HT, coppery-rose............... 230

30 Blance de Coubert, white............ 30

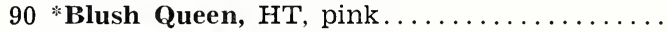

120 British Queen, $\mathrm{HT}$, white............ 220

110 *Cambria, Per, apricot to crimson.......... 10

$20 *$ Candeur Lyonnaise, HT, white........... 20

120 Captain Christy, HP, silver-pink . . . . . . . . 160

110 Captain F. Bald, HT, crimson.......... 100

160 Capt. F. S. H. Cant, HT, (1923), pink . . . . . 170

250 Charles K. Douglas, HT, scarlet. . . . . . . . 190

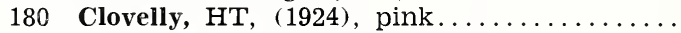

70 Col. O. Fitzgerald, HT, crimson.......... 40

120 Commonwealth, HT, deep pink......... 80

80 Coquette Des Alps, HN, white........... 60

50 Count. of Warwick, HT, lemon-yellow........

40 Covent Garden, HT, crimson........... 100

210 Crimson Queen, HT, Crimson........... 890

Crusader, HT, velvety-crimson......... 910

Duchess of Albany, HT, red.......... 50

270 Duchess de Brabrant, $\mathrm{T}$, pink . . . . . . . . . . 200

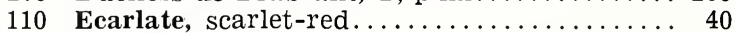

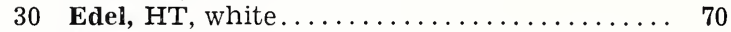

300 Edith Part, HT, rosy-red............ 200

910 Edward Mawley, HT, crimson ........... 650

20 *Emile Charles, Per, (1922), yellow ......... 30

Ethel Somerset, HT, shrimp-pink . . . . . . 180

$80 *$ Ethel James, HT, orange-pink......... 50

Etoile de France, HT, velvet-crimson. . . . . . 890

70 Eugene Furst, HP, crimson............. 40

60 Evelyn, HT, white to rose............ 110

Fischer Holmes, HP, crimson........... 10

60 *Florex, HT, (1927), salmon to orange...... 290 
N . 1 LOT A-Continued No. $1 \frac{1 / 2}{50}$

Frank W. Dunlop, HT, pink.......... 50

910 General McArthur, HT, scarlet.......... 850

270 General S. A. Janssen, HT, carmine....... 240

$50 *$ Georges Clemenceau, Per, orange......... 110

$150 *$ Gladys Holland, HT, buff-pink.......... 20

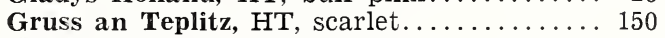

20 Hansa, HT, red to violet............. 10

50 *Harrison's Yellow, AB, gold-yellow........ 120

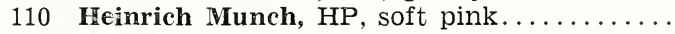

110 Helen Gould, HT, red................ 40

Helen Taft, $\mathrm{HT}$, pink................. 50

200 Henriette, HT, orange-crimson.......... 190

$160 *$ Herman Neuhoff, HT, (1923), red........ 260

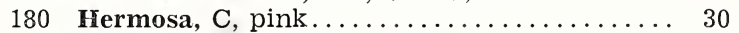

Hugh Dickson, HP, crimson.......... 70

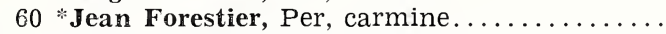

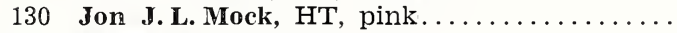

280 Killarney, shell pink. . . . . . . . . . . 310

Killarney Brilliant, $\mathrm{HT}$, crimson........ 40

Killarney Dbl. White, HT, white. . . . . . 590

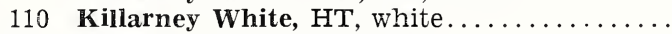

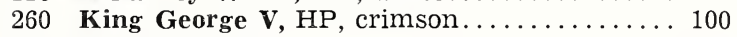

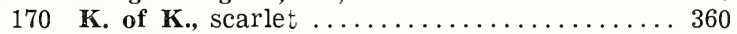

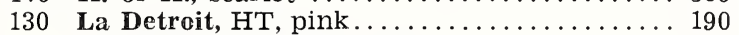

La Marne, $\mathrm{P}$, salmon-rose. . . . . . . . . . 150

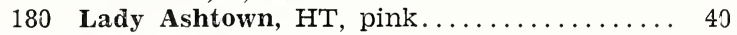

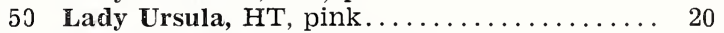

100 Laurent Carle, HT, carmine............. 170

$150 *$ Leader, HT, cerise . . . . . . . . . . . . . . 90

140 Mable Turner, HT, (1923), shell-pink. ..... 100

Maman Cochet, white............ 80

110 * Mary Count of Illchester, HT, carmine. . . . 140

260 Mme. Able Chatenay, HT, rose-salmon..... . 180

20 Mme. A. Chatenay, HT, crimson.......... 150

330 Mme. Caroline Testout, HT, pink . . . . . . . . . 360

70 Mme. Caroline Testout White, HT......... 60

110 * Mme. Emile Mayen, Per, (1924), gold. . . . . . . 220

70 Mme. $J u l e s$ Grolez, HT, rose.............

Mme. Leon Pain, HT, pink. . . . . . . . 30

70 Mme. Mallerin, HT, (1923), scarlet........ 80

120 Mme. Pernet-Ducher, HT, carmine. . . . . . . .

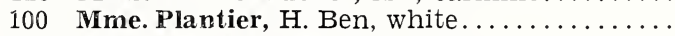

$60 *$ Mme. Ray Poincare, HT, yellow . . . . . . . . 30

20 Mr. P. L. Baudett, HT, carmine..........

Mrs. Chas. Lamplough, cream white....... 230

70 Mrs. C. W. Edwards, Per, (1924), carmine... 20

220 *Mrs. Farmer, Per, Indian yellow . . . . . . . . . 150

$220 *$ Mrs. Henry Bowles, HT, orange...........

$90 *$ Mrs. Henry Winnett, HT, crimson......... 20

*Mrs. H. R. Darlington, HT, creamy-white. . . 140

300 *Irs. John C. Mensing, HT, pink .......... 30

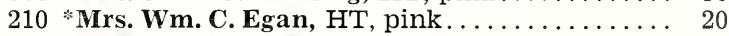

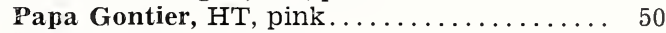

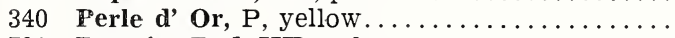

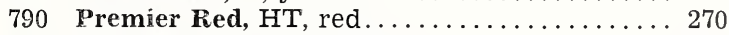

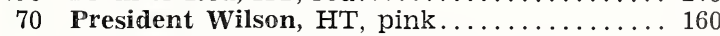

110 Prince de Bulgarie, HT, copper-pink . . . . . . . 180

120 Prince E. C. de Arenberg, HT, scarlet. . . . . . 170

$310 *$ Princess M. Jose, HT, (1924), orange. . . . . . .

Queen Beatrice, HT, pink. . . . . . . . . . 140

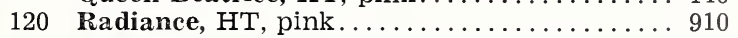

30 Red Letter Day, HT, scarlet-crimson..... . . . 360 
No. 1 LOT A-Continued

$100 *$ Rhea Reid, HT, velvety-red. . . . . . . . . . 80

50 Rose Dawn, HT, rose-flesh.............. 90 Iose Marie, HT, watermelon red......... 350 Souv. d Claud. Denoyel, HT, red......... 50

140 *Souv. d Mme. L. Crette, Per, (1923), gold . . . . 150

100 Souv. d Pres. Carnot, HT, white-flesh. . . . . . 160

180 Templar, HT, red..................

100 *otty's Rer, HT, scarlet............ 70

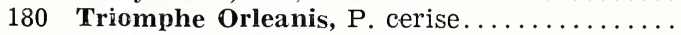

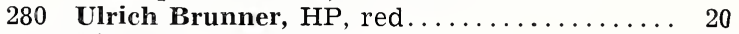

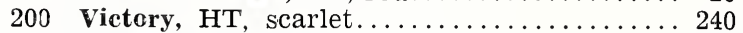

320 Wellsley, HT, pink . . . . . . . . . . . . . 220

William R. Smith, HT, cream to pink. .... 260

220 Winnie Davis, $\mathrm{HT}$, pink........... 70

\section{CLIMBERS}

110 Alida Lovett, WH, pink.

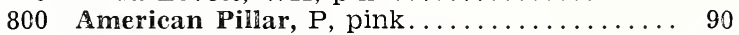

Aviateur Bleriot, WH, yellow. . . . . . . . 510

130 Banksia White, $B$, white.............. 30

240 Banksia Yellow, B, yellow.............. 100

$290 *$ Belle of Portugal, HG, pink ..........

Bess Lovett, W. red............... 20

Christine Wright, W, pink ........... 200

170 Cl. Able Chatenay, $\mathrm{HT}$, rose........... 80

200 Cl. Belle Siebrecht, HT, pink. . . . . . . .

40 Cl. Caroline Testout, HT, pink..........

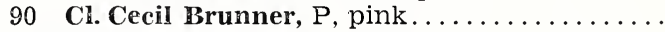

140 *Cl. Chat. de Clos Vougeot, red . . . . . . . . 110

210 Cl. Duchess of Wellington, HT, yellow . . . . . . 20

570 Cl. Edouard Herriott, HT, copper-red. . . . . . 120

100 Cl. Etoile de France, HT, red. . . . . . . . . . . 90

$570 *$ Cl. General McArthur, HT, red. . . . . . . . . .

40 Cl. Gruss an Teplitz, HT, scarlet. . . . . . . . .

$320 *$ Cl. Hadley, HT, crimson . . . . . . . . . .

$540 *$ Cl. Hoosier Beauty, HT, crimson. . . . . . . .

140 Cl. Jon J. L. Mock, HT, cherry-red..........

110 Cl. Winnie Davie, $\mathrm{HT}$, pink........... 40

Crimson Rambler, $\mathrm{P}$, crimson.......... 40

40 Derothy Perkins, HW, pink............

Dorothy Perkins White, white.......... 120

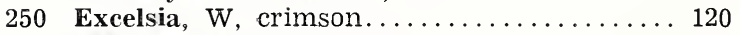

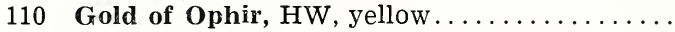

40 Heart of Gold, WH, crimson to yellow . . . . . 120

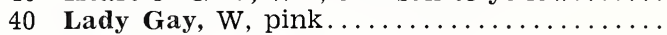

Rene M. Henriette, $\mathrm{N}$, crimson. . . . . . . . 100

320 Roserie, $M$, deep pink............... 290

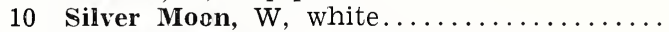

300 Tausendescheon, $P$, white to pink......... 110

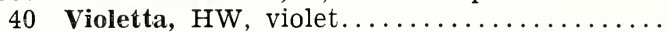

$$
\text { I. Ot B No. } 1 \frac{-30 c}{\text { No. } 1 / 2-20 c}
$$

*Arthur R. Goodwin, HT, copper.......... 60 Captain Hayward, HP, crimson.......... 130

10 Capt. Ronald Clerk, Per, (1923), scarlet..... 40

60 *hristine, HT, golden-yellow . . . . . . . 30

10 Clio, HP, flesh-pink. .............. 30

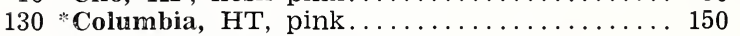

100 Columbia Silver, HTfi pink............

Duch. of Wellington, HT, yellow....... 200 
No. 1 LOT A-Continued

$190 *$ Eldorado, Per, golden-yellow. . . . . . . . . .

30 *E. P. H. Kingma, Per, apricot.......... 30

60 *vening Star, Per, golden-yellow . . . . . . . . 160

110 F. J. Grootendorst, R, red............ 24

60 * Florence L. Izzard, Per, (1923), yellow. . . . . 290

$30 *$ Fontanelle, HT, (1927), yellow............

150 Francisco Corbzera, Per, (1924), salmon..... 60

210 Frau Karl Druschki, HP, white.......... 24

$80 *$ Geisha, Per, orange-yellow............ 90

$10 *$ Gold Mine, HT, golden-yellow........... 30

$100 *$ Gorgeous, HT, orange-yellow . . . . . . . . . 110

$630 *$ Hadley, HT, crimson . . . . . . . . . . . . 250

210 Harry Kirk, T, yellow.............. 130

60 *Imperial Potentate, HT, pink. . . . . . . . . 120

10 Irene Thompson, HT, deep gold......... 70

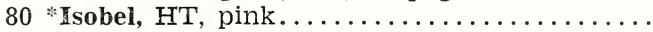

40 J. B. Clark, HP, scarlet............... 40

$90 *$ Joseph Baud, HT, orange-yellow.......... Jubilee, HP, crimson.............. 70

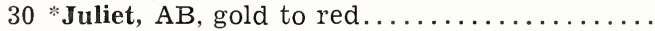

20 June Boyd, Per, (1924), salmon...........

$910 *$ Kaiserine A. Victoria, HT, white. . . . . . . . 890 Lady Hillingdon, HT, apricot.......... 240

110 *Lord Charlemont, HT, (1922), crimson.....

80 *Lord Lambourne, Per, (1925), yel.-car... . . . 100 * Los Angeles, HT, pink to coral. . . . . . . . 320

30 *ucinda, HT, (1927), bright rose........ 230

$20 *$ Lulu, HT, orange to copper............

60 * Marcia Stanhope, HT, (1924), white....... 50

70 *Maud Cummings, HT, (1923), pink to orange

30 *Miss Amelia Gude, HT, yellow........... 240

$60 *$ Mme. Alexandre Dreaux, Per, yellow. . . . . 50

*Mme. Butterfiy, HT, salmon........... 440

$910 *$ Mme. Edouard Herriott, HT, copper........ . Mme. E. van der Goes, HT, (1925), coral. ... 30

$70 *$ Mme. Henri Lustre, Per, (1924), red........ 60

90 Mime. Hippolyte Dumas, Per, (1924), flesh....

*Mme. L. Guenotte, HT, (1925), pink....... 220

*Mrs. Aaron Ward, HT, yellow........... 90

Mrs. A. R. Waddell, HT, saffron......... 210

$80 *$ Nederland, HT, deep red............. 20

20 Nona, Per, (1924), flame-pink.......... 130

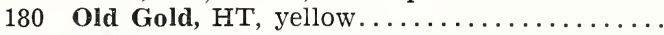

350 Ophelia, HT, salmon-pink........... 180

70 Ophelia Golden, HT, yellow.............

Ophelia Supreme, HT, rose-pink. . . . . . 270

40 Ophelia White, HT, white............ 130

$80 *$ Padre, HT, copper-scarlet.............

$60 *$ Pini Pearl, HT, (1924), rose-pink...........

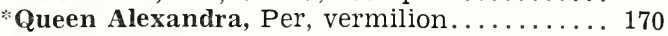

$30 *$ Roselandia, HT, yellow ............ 40

Rosette Delizy, T, yellow............ 30

40 * Solliden, Per, (1924), carmine........... 20

* Souv. d Claudius Pernet, Per, yellow ....... 310

100 *Souv. d Geo. Beckwith, HT, pink. . . . . . . . .

390 *Souv. d Georges Pernet, Per, red..........

60 * Souv. d Mme. Boullet, HT, yellow . . . . . . . . 130

910 *Sunburst, HT, yellow.............. 30

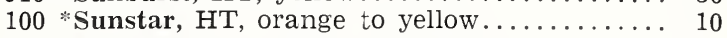

$170 *$ Surville, Per, (1924), Indian-red......... 70

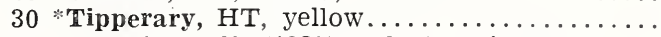

$140 *$ Vesuvius, HT, (1923), velvety-crimson..... 20 
No. 1 LOT B-Continued

$150 *$ William F. Dreer, HT, pink to yellow . . . . . 380

*Willowmere, Per, pink............ 30

\section{CLIMBERS}

$190 *$ Cl. Lady Hillingdon, T, apricot. . . . . . . . . 180

370 *Cl. Mrs. Aaron Ward, HT, Indian-yellow . . . . 30

$200 *$ Cl. Sunburst, HT, yellow . . . . . . . . . . . 120

*Duchess d' Auerstadt, T, yellow.......... 110

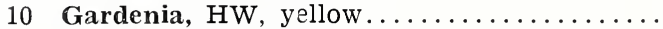

$60 *$ Wm. A. Richardson, N, yellow . . . . . . . 120

$$
\operatorname{IOt} C \begin{aligned}
& \text { No. } 1-40 c \\
& \text { No. } 11 / 2-30 c
\end{aligned}
$$

30 Betty Uprichard, HT. .............

40 *Elvira Armayo, Per, (1922), red..........

50 Empire Queen, HT, (1925), cerise........ 30

*Jules Tarbart, Per, salmon-pink........ 70

$30 *$ Ludwig Moller, HP, light yellow..........

170 *Mme. Paul Ollivary, HT, (1924), copper. . . . . 200

40 Mrs. L. Swisher, HT. . . . . . . . . . . . .

$30 *$ Odette Foussier, (1924), HT, orange........

*O. Jumyent, Per, yellow............ 20

W. E. Wallace, HT, (1922), golden........ 30

230 * Wilhelm Kordes, HT, (1922), golden . . . . . . . 240

$$
\text { Lot } D \text { No. } 1 \frac{11 / 2-40 c}{\text { No. }}
$$

$70 *$ Evelyn Murland, (1923), pink to coral......

$60 *$ Geo. H. Mackereth, HT, (1924), crimson.... . 20

$20 *$ Geraldine, HT, (1924), buff to pink....... 30

40 *Gooiland Beauty, Per, (1924), orange..... 30

$180 *$ Grillodale, HT, (1928), pink ........... 40

30 Sarah Van Fleet, HR, rose-pink..........

$60 *$ Westfield Star, HT, lemon-yellow......... 30

\section{CLIMBERS}

40 Cl. K. A. Victoria, HT, white..........

$40 *$ Cl. Sunstar, AB, yellow . . . . . . . . . 30

150 *Cl. Willowmere, Per, shrimp-pink ........ 50

* Marechal Neil, $\mathrm{N}$, yellow............. 150

Star of Persia, AB, yellow........... 10

$$
\text { Lot } \mathrm{H} \text { No. } 1-80 \mathrm{c}
$$

$26 *$ Angele Pernet, HT, (1924), orange.......... 


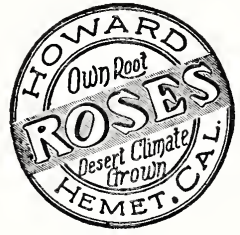

Terms-Our terms are cash, or satisfactory references before shipment. To established nurserymen, seedmen, florists and dealers known to us, or to those who have necessary rating. credit will be granted as follows: 60 days net f. o. b. Hemet, California; or for cash (with order or before shipment) $3 \%$ discount allowed; if paid in 10 days $2 \%$ allowed; if paid 30 days, $1 \%$ allowed. After 30 days no discount whatsoever.

To the Trade Only-Prices shown are for first-class stock of the grade named.

The use of printed stationery as protection is desirable. If it is not used, the purchaser should state clearly his position. We print only wholesale trade lists, and do not desire or solicit retail orders.

Substitution-We do not substitute unless instructed to do so, and then label true name. Customers will save time by naming a second choice, to be supplied only when first choice is sold out.

Pro Rata-As it is almost impossible to count Roses in the field when partly grown, there is a feature of uncertainty about all orders booked before digging. Consequently we reserve the right to fill such pro rata.

Genuineness-We exercise great care to keep all varieties true to name and will replace on proper proof, all stock that may prove otherwise, but it is mutually agreed that we shall not be liable for any amount greater than the original price of the stock. All quotations made subject to stock being unsold when order is received, or loss by any and all unavoidable causes. Freight shipments travel at risk of purchaser.

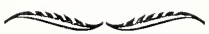

EXPRESS RATES FROM HEMET, Per 100 Ibs. to

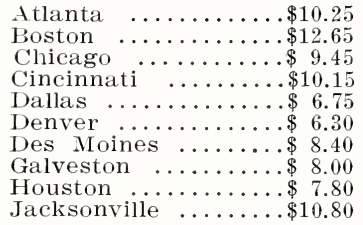

Kansas City ......\$7.80 Memphis ..........\$ 8.85 New Orleans .......\$ 9.00 New York .........\$12.15 Philadelphia $\ldots \ldots \ldots \$ 12.00$ Salt Lake .......\$ 4.40 St. Louis ........\$ 9.05 St. Paul ........... 99.45 Washington $\ldots \ldots \ldots \ldots 11.65$

Our No. 1 Roses weigh, packed, about 360 pounds pel 1000 plants. In large lots, say about 300 to 350 plants, these rates can be applied, but bales weighing less than 100 pounds cost more. 

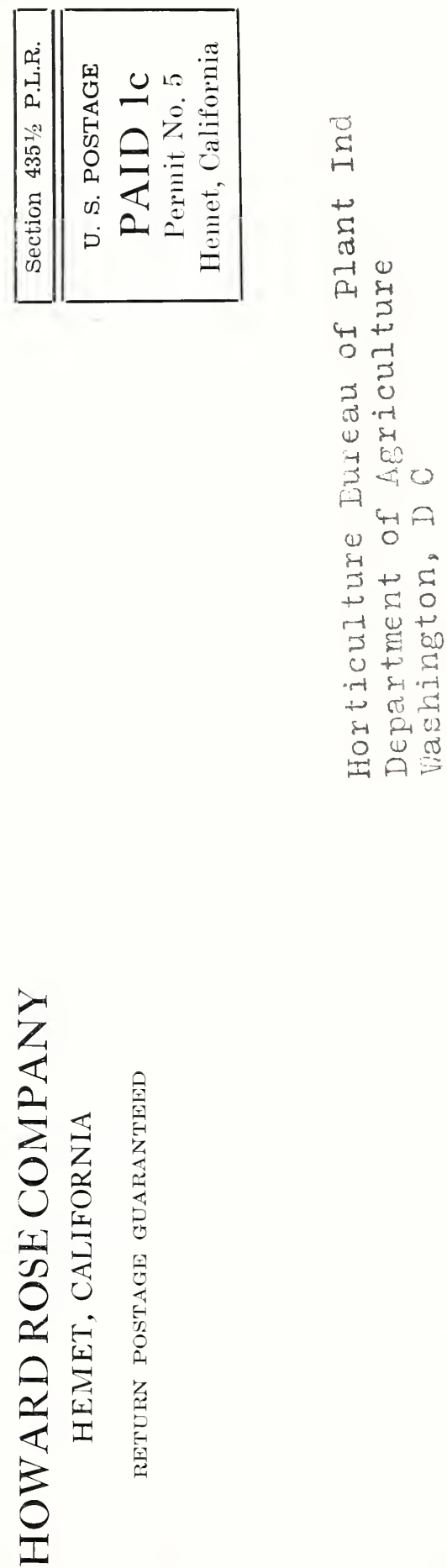